\title{
Abszeß im rechten Schläfelappen bei einem Linkshänder mit sensorischer Aphasie. ${ }^{1)}$ \\ Von
}

Priv.-Doz. Dr. Ernst Sträussler,

k. u. k. Regimentsarzt, Vorstand der psych.-neurol. Abteilung.

(Aus dem k. u. k. Garnisonsspital Nr. 11 in Prag [Kommandant: Oberstabsarzt Dr. Julius Schwarz].)

Mit 2 Textfiguren.

(Eingegangen am 18. April 1912.)

Die Linkshändigkeit, welche von jeher mit Rücksicht auf ihre Begleiterscheinung der rechtshirnigen Lokalisation der Sprachfunktion großes Interesse erweckte, ist in der letzten Zeit Gegenstand besonders intensiven Studiums geworden.

Ernst We ber ${ }^{2}$ ) beschäftigte sich in mehreren Abhandlungen mit den einschlägigen Fragen, Gau $\mathrm{pp}^{3}$ ) widmet dem Gegenstande eine ausführliche Studie, und in jüngster Zeit hat Stier ${ }^{4}$ ) in einem umfangreichen Buche mit umfassender Gründlichkeit die Bedeutung der Linkshändigkeit in allen Einzelheiten beleuchtet.

Unter den Ursachen für das Neuerwachen des Interesses an den Fragen, welche sich an die Linkshändigkeit des Menschen knüpfen, spielt keine geringe Rolle die in letzter Zeit zu einer gewissen Blüte gelangte „Doppelhandkultur“. Von den Bestrebungen dieser Bewegung, welche durch eine gleichmäßige Utbung und Ausbildung beider Hände neben "mechanischen und ökonomischen Vorteilen" den großen Gewinn einer Rückwirkung auf die Organisation des Gehirns, einer Verdoppelung der bisher beim Rechtshänder an die linke Hemisphäre geknüpften und auf diese Hemisphäre beschränkten höchsten psychischen Leistungen zu erzielen hofft, gibt bereits eine eigene Literatur Zeugnis.

Die übertriebenen Erwartungen, welche einzelne begeisterte Ver-

1) Das Präparat wurde in der „Wissenschaftlichen Gesellschaft deutscher Ärzte in Böhmen", Sitzung vom 17. November 1911, demonstriert.

2) Weber, Ernst, Das Schreiben als Ursache der einseitigen Lage des Sprachzentrums, Zentralbl. f. Physiol. 18. - Ursachen und Folgen der Rechtshändigkeit. Halle a. S. 1905.

3) Ga u pp, E., Über die Rechtshändigkeit des Menschen. Jena 1909.

4) Stier, Ewald, Untersuchungen über die Linkshändigkeit und die funktionellen Differenzen der Hirnhälften. Jena 1911. 
fechter an die Linkskultur knüpfen, drückte bereits Liep $\operatorname{man} n^{1}$ ) in einer interessanten kritischen Abhandlung auf ein sehr bescheidenes Maß herab; Stier gelangte auf Grund seiner Studien über die Linkshändigkeit und die funktionellen Differenzen der Hirnhälften zu einer ziemlich schroffen grundsätzlichen Ablehung der erwähnten Bestrebungen.

Der von mir beobachtete Fall soll einen kleinen Beitrag zur Lehre von der Linkshändigkeit liefern. Das Studium der bezüglichen Literatur ergibt eine relativ sehr geringe Zahl von Beobachtungen sensorischer Aphasie bei Linkshändern mit der entsprechenden Lokalisation des Herdes im rechten Schläfelappen; Fälle von motorischer Aphasie werden viel häufiger beobachtet.

Die Reinheit des klinischen Bildes - sensorische Aphasie ohne alle begleitende Lähmungserscheinungen - stempelt unsere Beobachtung zu einer besonderen Seltenheit. Diese Eigentümlichkeit des Falles hatte auch zu einer verhängnisvollen Fehldiagnose geführt.

Am 24. Sept. 1911 wurde der 22jährige, im 2. Dienstjahre stehende Landwehrinfanterist J. K. aus dem Landwehrmarodenhause in P. zum Garnisonsspital Nr. 11 in Prag transferiert.

Aus den bisher geführten Krankengeschichten und den ärztlichen Protokollen konnten folgende Daten zur Anamnese geschöpft werden: Der bisher vollkommen gesunde Mann wurde am 22. Juli 1911 anläßlich der Erntearbeiten aus seinem Garnisonsorte B. für 22 Tage in seine Heimat beurlaubt; auch gelegentlich der am 19. Juli vor dem Antritte des Urlaubs vorgenommenen ärztlichen Untersuchung wurde er als "gesund" befunden.

Als er am 12. Aug. wieder in seinen Garnisonsort einrückte, klagte er über Kopfschmerzen, allgemeine Mattigkeit, Schwindelgefühl und Appetitmangel und es wurde eruiert, daß er am 27. Aug. in seiner Heimat bei intensiver Sonnenglut erkrankt und unter Erscheinungen von Kopfschmerz, Schwindel, Erbre. chen, Delirien, 2 Tage andauernder Bew u Btlosigkeit und Fieber darniedergelegen sei. Die Diagnose des behandelnden Zivilarztes hatte auf Hirnhautentzündung infolge Insolation gelautet.

Bis zum 22. Aug. wurde er im Landwehrmarodenhause in B. beobachtet, ohne daß irgendwelche pathologische Symptome in Erscheinung getreten wären; auch die weitere Beobachtung im Landwehrmarodenhause in P. bis zum 31. Aug. förderte keine auffallenden Erscheinungen zutage. Er wurde daher am 31. Aug. in seine Garnison zurückgesendet mit der Bestimmung, beim Wachdetachement des zu den Manövern abmarschierten Regiments leichte Dienste zu versehen.

Er beklagte sich aber von neuem über Kopfschmerzen, Mattigkeit und Schlaflosigkeit, und da die Beschwerden entsprechenden medikamentösen Maßnahmen nicht wichen, vielmehr auch noch ,verwirrtes Sprechen" hinzutrat, wurde am 8. Sept. die Wiederaufnahme des Kranken ins Landwehrmarondehaus in $\mathrm{P}$. veranlaßt.

Der hier am 8. Sept. aufgenommene Status praesens enthält an bemerkenswerten Details: Sehr apathisch, sehr blaß; fieberfrei. Temperatur $36,4^{\circ}$. Puls 60. Spricht sehr wenig, auf Fragen antwortet er kurz, aber gegenwärtig klar.

1) Liepmann, Über die wissenschaftlichen Grundlagen der sog. Linkskultur. Deutsche med. Wochenschr. 1911. 
Schon in den nächsten Tagen trat aber das Bild einer schweren cerebralen Erkrankung zutage: Somnolenz, Erbrechen, starke Kopfschmerzen, welche in die Stirngegend lokalisiert wurden, Anisokorie, Herabsetzung der Sehschärfe; direkte und konsensuelle Lichtreaktion weniger ausgiebig und träger. Die Untersuchung des Augenhintergrundes ergab eine Schwellung des Seh. nervenkopfes mit unscharfer Begrenzung der Papille, Venen stark dilatiert, pulsierend, Arterien etwas verschmälert. Sehschärfe: 6/12 beiderseits.

Im Nervenbefund waren sonst gar keine pathologischen Erschei. nungen nachweisbar. Der Gehörapparat erwies sich als normal.

Im weiteren Verlaufe der Beobachtung machte sich ein auffallender Kräfteverfall geltend, es bestand Schlafsucht und Somnolenz; sehr häufig stellte sich Erbrechen ein. In der Krankengeschichte findet sich außerdem die besonders beachtenswerte Bemerkung: ,Spricht zeitweilig verwirrt".

Die Pulsfrequenz bewegte sich um 60 Pulsschläge in der Minute, die Temperatur zeigte keine Steigerung.

Bei der Aufnahme ins Garnisonsspital in die Beobachtung des Verf. am 24. Sept. nachmittags ist er stark somnolent, körperlich hochgradig herabgekommen, bietet aber somatisch außer einem etwa kronengroßen, granulierenden, leicht sezernierenden, mit einer Borke bedeckten Substanzverlust am Nacken, keine pathologischen Erscheinungen.

Der Nerven befund ergibt an pathologischen Erscheinungen: Beide Pupillen relativ weit, l $>$ r. Lichtreaktion der linken Pupille wenig ausgiebig und träge, der rechten etwas besser. Konvergenzreaktion wegen Benommenheit des Pat. nicht sicher zu prüfen. Bauchreflexe nicht auslösbar. Positiver Kernig. Große Empfindlichkeit am ganzen Körper, Nackenschmerzen. Temperatur 37,2, Puls 62.

Das Examen, welches mit Rücksicht auf die Benommenheit des Pat. sehr erschwert ist, ergibt eine Störung des Sprachverständnisses und Paraphasie.

Die am 26. Sept. durch Dr. Löwenstein (Assistent der deutschen Augenklinik) vorgenommene ophthalmoskopische Untersuchung ergibt: Beide Papillen stark angeschwollen, rechts stärker (3 Dioptrien), gerötet, unscharf begrenzt. Venen stark erweitert, geschlängelt, Arterien von normalem Kaliber.

In den nächsten Tagen wiederholte sich öfters das Erbrechen, es traten in unregelmäßiger Weise Temperatursteigerungen bis auf $37,8^{\circ}$ auf, die Pulsfrequenz blieb etwas retardiert, die Sehschärfe nahm sichtlich ab, der Allgemeinzustand besserte sich aber so, daB am 4. Okt. eine genauere Untersuchung vorgenommen werden konnte.

Dieselbe ergab: Linke Pupille weiter als die rechte, Lichtreaktion (direkt und konsensuell) beiderseits träger und weniger ausgiebig, Konvergenzreaktion intakt. Bulbusbewegungen ohne Störung, Hornhautsensibilität ungestört. Facialisinnervation beiderseits gleich, Zunge wird gerade vorgestreckt. Grobe motorische Kraft in beiden oberen und unteren Extremitäten gering, aber ohne Differenzen. Biceps- und Tricepsreflexe, Periostreflexe an den oberen Extremitäten beiderseits gleich, in normaler Stärke. Bauchhautreflexe und Cremasterreflexe nicht auslösbar. Patellarreflexe fehlen, Achillessehnenreflexe vorhanden, kein Babinski. Oberflächliche und tiefe Sensibilität ohne Störung, stereognostischer Sinn intakt (die durch die später zu beschreibende Aphasie bedingten Fehler konnten ausgeschaltet werden), keine Ataxie, keine Gleichgewichtsstörung, Ker nig noch vorhanden; Gehörorgan ohne auffallende Erscheinungen, Hörschärfe normal. Sehschärfe auf Fingerzählen in einem Meter herabgesetzt. Keine wesentliche Einschränkung des Gesichtsfeldes, jedenfalls keine Hemianopsie.

Das Examen hinsichtlich der schọn früher fast bei jeder Untersuchung in 
verschieden starkem Maße zutage getretenen A phasie ergibt: S pontansprache: Mitunter geht ein kurzes Gespräch ohne jede Störung vonstatten, er drückt kurze Wünsche ganz korrekt aus. Dann mischen sich vereinzelte paraphatische Fehl. griffe in seine Äußerungen; er verlangt z. B. „rote“ Milch statt ,warme“ Milch; nach seinem Alter gefragt, meint er: „Ich erinnere mich nicht, wie lange ich alt bin."

Das Bezeichnen vorgezeigter Gegenstände erfolgt teilweise richtig, ziemlich häufig aber falsch; dabei deutet er den Gebrauch des betreffenden Gegenstandes durch Gesten richtig an. Einen "Schlüssel" bezeichnet er als „Birne“, auf die Frage nach dem Zwecke des Gegenstandes macht er mit der rechten Hand die Bewegung des Aufsperrens und meint: „Man schlüsselt damit" (Klí̌í se stim) - der Pat. spricht tschechisch, das Verbum ist im Tschechischen ebenso wenig gebräuchlich wie im Deutschen. Bei Fehlreaktionen agnosziert er aus mehreren ihm vorgesagten Worten oft, aber nicht immer die richtige Bezeichnung; die einzelnen Worte spricht er auch richtig nach. Zum Nachsprechen komplizierterer Worte oder von Sätzen ist er nicht zu bringen.

Seinen Namen schreibt er über Aufforderung richtig auf, bedient sich dabei der rechten Hand, statt des Wortes ,stary" schreibt er ,zarel“. Eine Leseprüfung ist schon mit Rücksicht auf seine Sehstörung sehr schwierig; er ist nicht dazu zu bringen, auch die größten Buchstaben zu lesen.

Zeitweilig läßt er an ihn ergangene Aufforderungen zu einfachen Handlungen unbeachtet, während er später den gleichen Aufforderungen Folge leistet; er zeigt die Zunge, schließt die Augen, hebt den Arm; nicht selten erfolgt aber eine verkehrte Handlung, offenbar infolge gestörten Sprachverständnisses.

In den nächsten Tagen wird er wieder benommener. Das Examen ergibt, daß er beim Bezeichnen von Gegenständen ein genanntes Wort beim Vorzeigen von weiteren Gegenständen wiederholt. Ebenso perseveriert er in Handlungen, die er über Aufforderung ausgeführt hat.

Im weiteren Verlaufe steigt die Pulsfrequenz, sie hält sich zwischen 80 und 90, die Temperatur zeigt, wie früher, intermittierend leichte Steigerungen: das subjektive Befinden und die Benommenheit machen noch einige beträchtliche Schwankungen durch, der Befund hinsichtlich des Nervenzustandes und der Aphasie bleibt im wesentlichen stationär; es treten keinerlei Herderscheinungen hinzu. Eine neuerliche, am 18. Okt. vorgenommene Untersuchung des Augenhintergr undes ergibt: Schwellung beider Papillen, unscharfe Begrenzung, in der Peripherie streifenförmige, radiäre Blutungen, stark erweiterte geschlängelte Venen, ober- und unterhalb der Papille Exsudatherde mit Flecken von weißgrauer atrophischer Verfärbung.

Unter Zunahme der Benommenheit, Steigerung der Pulsfrequenz und Temperaturzunahme auf $39^{\circ}$ tritt am 30. Okt. der Exitus ein.

In Kürze zusammengefaßt stellt sich der beobachtete Krankheitsfall wie folgt dar: Ein 22 jähriger Mann, welcher fast zwei Jahre im Dienste steht und niemals irgendwelche Krankheitserscheinungen dargeboten hatte, erkrankt während einer Beurlaubung in seiner Heimat am 27. Juli plötzlich unter schweren, mit Fieber einhergehenden cerebralen Symptomen, welche den behandelnden Arzt veranlassen, eine Meningitis anzunehmen. Der Zustand bessert sich nach etwa zwei Wochen derart, daß der Mann sich wieder zum Dienste stellt. Er klagt über Kopfschmerzen, Schwindel und Mattigkeit, bis Anfang August können aber durch die ärztliche Beobachtung keine objektiven Krank: 
heitserscheinungen nachgewiesen werden. Gegen Mitte August - es waren indes Erbrechen und eine Pulsverlangsamung zu der subjektiven Erscheinung des heftigen Kopfschmerzes hinzugetreten - ergibt die Untersuchung des Augenhintergrundes eine Stauungspapille. Außer geringen Pupillenstörungen sind keinerlei nervöse Störungen nachweisbar, dagegen wird von zeitweiligem „verwirrten Sprechen“ berichtet. Als der Kranke dann in unsere Beobachtung kommt, bietet er neben allgemeinen Erscheinungen von Hirndruck, Andeutungen von meningitischen Symptomen und leichten Temperatursteigerungen eine sensorische A phasie. Im weiteren Verlaufe steht dann die Aphasie, welche sich durch eine Störung des Sprachverständnisses, verbale Paraphasie, Paragraphie und verbale- und Handlungsperseveration charakterisiert, im Vordergrund des Symptomenbildes. Es fehlt bis zu dem etwa 3 Monate nach dem Krankheitsbeginn eingetretenen Exitus sonst jedwede andere Herderscheinung.

In den diagnostischen Erwägungen wurde eine Zeitlang mit der Möglichkeit einer Meningitis gerechnet, wobei das Herdsymptom der sensorischen Aphasie als Drucksymptom aufgefaßt werden mußte. Schon mit Rücksicht auf die Seltenheit einer solchen Erscheinung konnte die Diagnose nicht befriedigen. Als die meningitischen Erscheinungen im weiteren Verlaufe der Krankheit weder eine Bereicherung noch auch eine Steigerung erfuhren, die sensorische Aphasie sich als isoliertes und dauerndes Herdsymptom erwies, welches das Symptomenbild beherrschte, wurde die Annahme einer Meningitis fallen gelassen, um so mehr als die relativ starke Schwellung der Papillen dieser Diagnose keineswegs günstig schien.

Die in Betracht kommenden Möglichkeiten engten sich nun sehr ein; als das Nächstliegende erschien jetzt ein raumbeschränkender Prozeß mit dem Sitze im linken Schläfelappen. „Tumor" stand selbstverständlich im Vordergrund der Erwägungen. Das akute Einsetzen der Krankheitssymptome und die beobachteten unregelmäßigen Temperatursteigerungen unterstützten jedoch die Vermutung, daß es sich um einen Absceß handeln könnte. Der erwähnte Substanzverlust am Nacken, welcher als Rest eines Furunkels imponierte, ließ daran denken, hier den Ursprungsort für einen metastatischen Absceß zu suchen. Mit Rücksicht auf die Intaktheit des Gehörapparates fehlte jeder Anhaltspunkt für die Annahme einer otogenen Abscedierung.

Dieses Endergebnis des diagnostischen Kalkuls drängte selbstverständlich dazu, in einem operativen Eingriff das Heil für den Patienten und die Lösung des diagnostischen Problems zu suchen. Die Möglichkeit, den pathologischen Prozeß auf einen relativ engen Gehirnbezirk zu lokalisieren und die Vermutung auf einen Gehirnabsceß gestatteten ja 
an einen operativen Eingriff die denkbar günstigsten Erwartungen zu knüpfen.

Unsere Absichten, den Kranken dem Chirurgen zuzuführen, mußten aber dem Widerstande der Angehörigen, welche die Zulassung jedes Eingriffes, auch einer Hirnpunktion, verweigerten, weichen. Die Autopsie brachte nun eine große Utberraschung. Unsere diagnostischen Erwägungen hatten prinzipiell zu einem richtigen Resultat geführt praktisch genommen standen wir vor einer Fehldiagnose: der im lin ken Schläfelappen vermutete $A b s c e ß$ fand sich im rechten Schläfelappen. Eine auf den linken Schläfelappen beschränkte Explorativpunktion hätte ein vollkommen negatives Ergebnis gehabt.

Es wurde bloß die Sektion des Schädels vorgenommen. An der Konvexität des Gehirns prägte sich in einer hochgradigen Abplattung

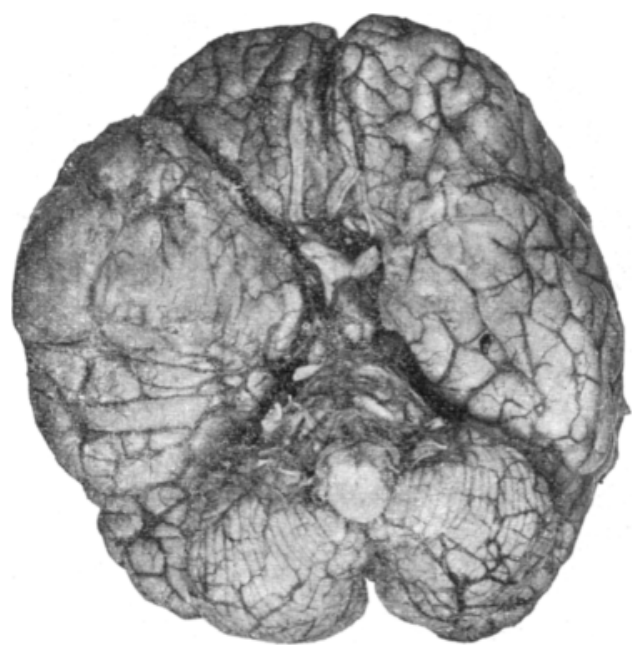

Fig. 1.

der Gehirnwindungen sehr deutlich der gesteigerte Gehirndruck aus. Bei der Herausnahme des Cerebrum zeigt es sich, daß der rechte Schläfelappen an seiner Basis in einer etwa hellergroßen Ausdehnung an die harte Hirnhaut durch leicht zu lösende Adhäsionen angelötet ist.

Der rechte Schläfelappen ist gegen die Basis und nach vorne zu sehr stark vorgewölbt, imponiert als hühnereigroße, von einer dünnen Schicht von Gehirnsubstanz bedeckte schwappende Geschwulst. Das Gehirn wurde in toto in Formol aufbewahrt.

Die Aufmeißelung der Schädelbasis, der Pyramide und des Processus mastoideus, hatte bezüglich eines Eiterungsprozesses ein negatives Ergebnis.

Fig. 1 zeigt in der Aufnahme des in Formol gehärteten Gehirns, 
von der Basis aus, die starke Vorwölbung des rechten Schläfelappens; dessen Spitze überragt die Spitze des linken Schläfelappens' um $2,5 \mathrm{~cm}$, die Formation der Oberfläche nach Furchen und Windungen erscheint im wesentlichen erhalten.

Ein durch das Gehirn 1,2 cm von der Spitze des linken Schläfelappens angelegter Frontalschnitt führte in die Mitte einer etwa hühnereigroßen, teils mit flüssigem, teils mit schmierig weichem gelblich-grünen Eiter gefüllten, von einer ziemlich dicken pyogenen Membran versehenen Absceßhöhle. Fig. 2 gibt die Photographie der hinteren Schnittfläche wieder.

Weiter gegen die Spitze des Schläfelappens zu geführte Frontal-



Fig. 2

schnitte ergeben, daß sich hier noch zwei kleinere, von einer pyogenen Membran umgebene, isolierte Abscesse finden.

Die Betrachtung der Frontalschnitte ergibt, daß die Abscesse etwa die vorderen zwei Drittel des Schläfelappens einnehmen; die Spitze desselben erscheint vollkommen destruiert. Die große Absceßhöhle, deren längster Durchmesser frontal gestellt ist, nimmt die basalen Teile des Schläfelappens ein; die äußere Wand wird durch die auf etwa $2 \mathrm{~mm}$ verdünnte enorm ausgedehnte Rinde der dritten Schläfewindung gebildet. Die Absceßhöhle ragt aber derart in das Marklager der zweiten und ersten Schläfewindung hinein, daß wohl auch diese in ihren vorderen zwei Dritteln ausgeschaltet erscheinen; überdies ist das Marklager der 2. Schläfewindung im ganzen Bereiche des Abscesses durch eine Erweichung in schwere Mitleidenschaft gezogen.

Die ganze rechte Hemisphäre ist entsprechend der Ausdehnung der Abscesse sehr stark geschwollen und drängt die linke Hemisphäre zur Seite.

Die sorgfältigste Durchforschung des linken Schläfelappens läßt nicht die geringsten Veränderungen erkennen.

Bei der mikroskopischen Untersuchung des rechten Schläfe- 
lappens ergibt sich hinsichtlich der Abscesse das gewöhnliche Bild. Hervorzuheben ist, daß die pyogene Membran eine reichliche Bindegewebsentwicklung aufweist und das aus dem histologischen Befunde zu erschließende Alter der Abscesse mit dem klinischen Verlaufe im Einklange steht.

Der makroskopisch als Erweichung imponierende Herd ist dem mikroskopischen Bilde nach durch einen Zerfall des nervösen Gewebes hervorgerufen, welcher mit einer intensiven, nichteitrigen Entzündung einhergeht.

Sowohl im Aufstrichpräparate des Eiters, als auch in den Schnitten finden sich a usschließlich grampositive Kokken zu zweien und in Häufchen angeordnet, zum Teil frei, meistens in Zellen eingeschlossen. Herr Professor Ghon, welcher die Güte hatte, die Präparate durchzusehen, erklärt, daß man, auch ohne Kontrolle durch das Kulturverfahren, berechtigt sei, nach dem morphologischen und färberischen Verhalten die vorhandenen Bakterien als der Gattung Staphylococcus zugehörig anzusprechen. Aus diesem Befunde könne man mit größter Wahrscheinlichkeit auf einen ätiologischen $\mathrm{Zusammen-}$ hang der Hirnabscesse mit dem Furunkel am Nacken schlieBen, da ja als Erreger für Furunkel der Staphylococcus pyogenes aureus in erster Linie in Betracht kommt.

Die hinteren Teile des rechten Schläfelappens erweisen sich auch bei der mikroskopischen Untersuchung als intakt.

Angesichts des Sektionsbefundes lag nichts näher, als an eine Lin kshändigkeit des Kranken zu denken. Wohl hatten wir selbst beobachtet, daß er mit der rechten Hand schrieb, daß er den Löffel beim Essen mit der rechten Hand führte und daß er sich bei der Demonstration des Gebrauches der vorgezeigten Gegenstände, die er paraphatisch bezeichnete, der rechten Hand bediente. Diese Momente dürften auch bis zu einem gewissen Grade als Entschuldigung dafür gelten können, daß wir bei Lebzeiten des Kranken die Möglichkeit einer Linkshändigkeit und der Erkrankung des rechten Schläfelappens ganz und gar außer Kombination ließen. Die Studien über Linkshändigkeit, insbesondere die Untersuchungen von Stier haben gezeigt, daß alle die genannten Hantierungen auch bei einem Linkshänder mit vornehmlicher Rechtshirnigkeit durch die Erziehung auf die rechte Hand gleichsam übertragen werden können; bezüglich des Schreibens gilt dies ja beinahe als Regel.

Die beim Vater des Mannes nachträglich gepflogenen Erhebungen ergaben nun tatsächlich, daß dieser Linkshänder war. Er galt zu Hause in der Kindheit als solcher; nach Angabe des Vaters lernte er wohl mit der rechten Hand schreiben und essen, bei allen Arbeiten, welche einer besonderen Kraft oder Geschicklichkeit bedurften, - 
er hatte kein Handwerk gelernt und war bloß Tagarbeiter - habe er sich der linken Hand bedient; der Vater führte besonders an, daß er beim Steinewerfen stets die linke Hand vorzog. Auch beim Truppenkörper des Patienten wurde hinsichtlich dessen Linkshändigkeit nachgeforscht und eruiert, daß er beim Schuhputzen und Brotschneiden mit Vorliebe die linke Hand benützte.

Wenn wir die von Stier aus dem großen Untersuchungsmaterial der Linkshänder der deutschen Armee geschöpften Tabellen über die diagnostische Bedeutung gewisser von ihm zur Prüfung der Linkshändigkeit gewählter Tätigkeiten zu Rate ziehen, so können wir die Bevorzugung der linken Hand beim Schuhputzen, Brotschneiden und Steinewerfen als ganz verläßliche Kriterien für die Linkshändigkeit betrachten. Wir können es also als erwiesen betrachten, daß der Patient ein Linkshänder war, dessen Sprachzentren ihren Sitz in der rechten Hemisphäre hatten.

Wir sind daher der Notwendigkeit enthoben, unsere Beobachtung unter die Fälle von sogenannter ,gekreuzter Aphasie" einzureihen; ein derartiger, allen Umständen nach ziemlich einwandfreier Fall von „Rechtshirnigkeit bei einem Rechtshänder" mit sensorischer Aphasie wurde erst vor kurzem von Lewandowsky ${ }^{1}$ ) publiziert und die Ubbersicht der Literatur hinsichtlich einer durch rechtsseitige Herde hervorgerufenen sensorischen Aphasie ergibt die bemerkenswerte und auffallende Tatsache, daß es sich in mindesten der Hälfte derartiger Beobachtungen nach Angabe der Autoren um Rechtshänder handelte.

Stier, welchem die Beobachtung Lewandowkys noch nicht bekannt war, verhält sich gegenüber der Annahme einer solchen Umkehrung der Bedeutung der beiden Hemisphären sehr skeptisch. Zu den bisher gegen eine derartige Auffassung geltend gemachten Argumenten bringt er ein neues sehr beachtenswertes Moment bei: Es sei noch niemals eine derartige Umkehrung bei einem anscheinenden Linkshänder beobachtet worden in dem Sinne, daß die für die Hand und für die Sprache bevorzugten Hirnhälften sich nicht deckten; wenn tatsächlich solche Ausnahmen vorkommen, dann müßten sie außer bei Rechtshändern doch auch wenigstens ein oder das andere Mal bei einem Linkshänder beobachtet worden sein. Die ihren eigenen oder fremden Angaben nach als linkshändig bezeichneten Menschen seien auch wirklich in der Regel als Linkshänder zu betrachten; da die als rechtshändig erscheinenden oder so bezeichneten dagegen durch Erziehung zu scheinbaren Rechtshändern aus linkshändig veranlagten Menschen geworden sind, so sei es direkt zu erwarten, daß bei man-

1) Lewandowsky, Rechtshirnigkeit bei einem Rechtshänder. .Zeitschr. f. d. ges. Neurol. u. Psych. 4. 1911. 
chen dieser scheinbaren Rechtshändigen auch das Sprachzentrum im rechten Gehirn liege, da es sich eben um Linkshänder handle. Es erscheine aber unwahrscheinlich, daß jemals ein notorischer Linkshänder mit einem im linken Gehirn lokalisierten Sprachzentrum gefunden werden sollte, da die Erziehung wohl Linkshänder zu Rechtshändern mache, nicht aber umgekehrt Rechtshänder zu Linkshändern.

Stier meint daher, daß die Annahme einer einheitlichen Lokalisation der Sprachzentren in der auch sonst dominierenden, ,superioren" Hirnhälfte die allein richtige ist und es sich in den dieser Anschauung scheinbar widersprechenden Beobachtungen um Linkshänder handelte, wenn nicht etwa eine Läsion in der linken Hemisphäre übersehen wurde. Eine in jüngster Zeit von Kurt Mendel') publizierte Beobachtung von motorischer Aphasie bei einem Rechtshänder durch einen rechtshirnigen Herd hervorgerufen, scheint freilich die absolute Gültigkeit der Stierschen Argumentation endgültig zu Falle zu bringen. Bezüglich der sensorischen Aphasie legt aber schon A. Pick ${ }^{2}$ ) mit Recht ein großes Gewicht auf die Mitwirkung des rechten Schläfelappens beim Sprachverständnis, so daß die durch Läsion des rechten Schläfelappens beim Rechtshänder entstehende sensorische Aphasie der Rätselhaftigkeit entkleidet erscheint.

Unser Fall beweist aber von neuem, daß ge nerell bei Linkshändern der Sitz der Sprachzentren in der rechten Hemisphäre zu suchen sei und daß diese Lokalisation auch dann zu Recht bestehen bleibt, wenn das Schreiben mit der rechten Hand erlernt und die Umgewöhnung auch für eine Anzahl alltäglicher Hantierungen auf die rechte Hand durch die Erziehung erzielt wurde. Aus unserer Beobachtung läßt sich also keine Aufmunterung für die Bestrebungen der Zweihandkultur schöpfen; es machten sich auch keine Anzeichen einer Restitution der Sprachfunktion während der drei Monate andauernden Erkrankung bemerkbar, trotzdem ja die sensorische Aphasie im allgemeinen schon in kürzerer Zeit einer Restitution zugänglich zu sein pflegt. Freilich muß berücksichtigt werden, daß hier der gesteigerte Hirndruck die Substitution durch andere Hirnteile - sowohl durch angrenzende Partien als durch homologe Teile der anderen Hemisphäre schwer beeinträchtigen konnte.

Die der sensorischen Aphasie eigentümlichen günstigen Restitutionsbedingungen mögen zum Teil die Tatsache erklären, daß (nach der Zusammenstellung Moutiers) ${ }^{3}$ ) in der Literatur viel mehr Fälle von motorischer Aphasie, durch rechtsseitige Herde hervorgerufen, existieren

1) Mendel, Kurt, Über Rechtshirnigkeit bei Rechtshändern. Neurol. Centralbl. 1912, Nr. 3.

2) Pick, A., Über das Sprachverständnis. Leipzig 1909.

3) Moutier, L'aphasie de Broca. Paris 1908. 
als Fälle sensorischer Aphasie. Ob auch etwa die von Stier besonders hervorgehobene und mit einleuchtenden Argumenten belegte Anschauung, daß das phylogenetisch ältere sensorische Sprachzentrum trotz der funktionellen Differenzen zwischen dem rechten und linken Gehirn vielmehr als das phylogenetisch jüngere motorische doppelseitig angelegt ist, für die Erklärung der relativen Seltenheit der publizierten sensorischen Aphasien durch rechtsseitigen Herd bei Linkshändern verwertet werden kann, mag dahingestellt bleiben.

Außerdem kommt aber sicher der Umstand in Betracht, daß sensorische Aphasien viel eher als motorische ohne sonstige Herderscheinungen bestehen können; in diesen Fällen wird also klinisch die Diagnose der Seite des Sitzes der Läsion selten gestellt werden können.

Der Mangel an anderen Herderscheinungen neben der Aphasie brachte es auch in unserem Falle mit sich, daß uns der rechtsseitige Sitz des Herdes bei Lebzeiten des Kranken vollkommen verborgen blieb. Ohne Autopsie wäre diese Lokalisation des Herdes niemals zu unserer Kenntnis gelangt. Dieses Moment illustriert die klinische Bedeutung unserer Beobachtung. Die uns nachträglich vom Vater des Kranken gegebenen anamnestischen Daten bestätigten unsere Vermutung, da/. der Substanzverlust am Nacken von einem Furunkel herrührte; derselbe war einige Tage vor dem Ausbruche der Erkrankung zur Entwicklung gekommen, so daß die Annahme eines ätiologischen Zusammenhanges des Gehirnabscesses mit dem Furunkel vollkommen gerechtfertigt erscheint. 